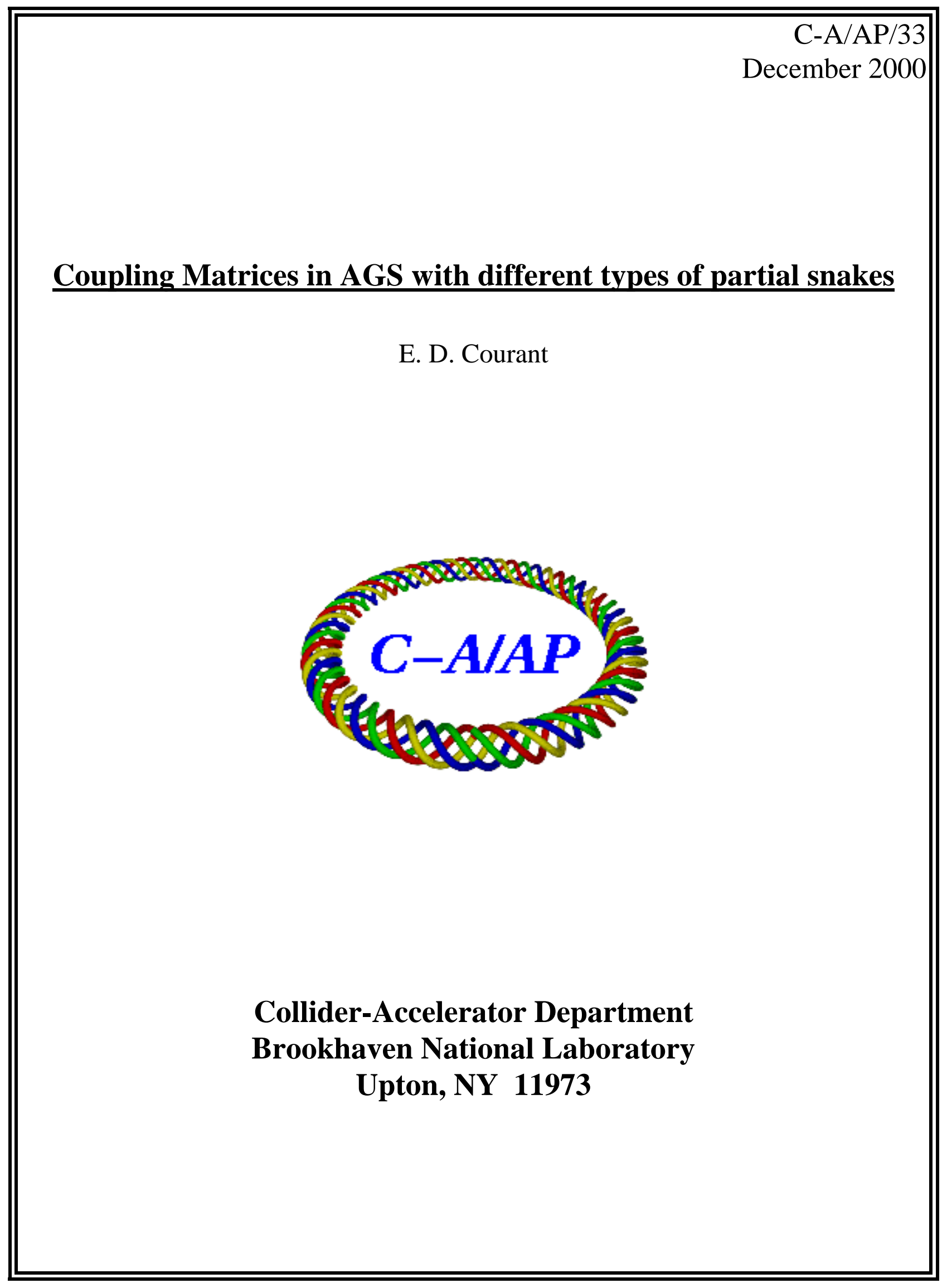




\title{
Coupling Matrices in AGS with different types of partial snakes
}

\author{
E D Courant \\ December 5, 2000
}

The solenoid partial snake in the AGS produces an irreducible amount of coupling between the horizontal and vertical betatron oscillation modes. Therefore alternate partial-snake schemes have been proposed: (a) a helix (with correction dipoles at its ends); (b) a sequence of four deflecting magnets with field orientations at various angles

Matrices for the AGS with a partial snake have been computed using the MATHEMATICA computer program. The helical matrices are evaluated using an algorithm previously formulated ${ }^{3}$, which takes into account the solenoidal fields and nonlinearities dictated by Maxwell's equations in a helical magnet, and the end effects due to the orbit excursions away from the axis of the helix. The partial snake is assumed to be in the $\mathrm{C} 10$ straight section of the AGS, and the matrices, with or without snakes, are evaluated at the upstream end of $\mathrm{C} 10$.We neglect sextupole and other multipole fields in the dipoles and in the helical magnet.

In these calculations we assume the following parameters:

AGS without snakes: $v_{\mathrm{x}}=8.698, v_{\mathrm{y}}=8.749$ (about .05 units away from coupling resonance). rotators.

Strength of partial snakes (solenoidal, helical, or dipole) adjusted to make $5 \%$ snakes, e.g. $9^{\circ}$ spin

Solenoid: length $2.5 \mathrm{~m}$; field strength ramping proportional to main field: $0.167 \mathrm{~T}$ at injection (1.5 $\mathrm{GeV}$ ) to $1.825 \mathrm{~T}$ at $\gamma \mathrm{G}=46.5$ (transfer to RHIC).

Helix as in ref. 1: Helix length $1.5 \mathrm{~m}$, compensating magnets $0.3 \mathrm{~m}$ each. Helix field ramping from $1.25 \mathrm{~T}$ at injection to $1.61 \mathrm{~T}$ at transfer.

4-magnet snake: magnet lengths $0.41,0.66,0.66,0.41 \mathrm{~m}$; field direction (from vertical) $-60^{\circ}$, $147^{\circ}, 33^{\circ},-120^{\circ}$; field ramping from $1.5 \mathrm{~T}$ (injection) to $1.94 \mathrm{~T}$ (transfer)

In each case the coupling strength is measured by two quantities: the "minimum tune separation", and the "coupling angle". Both of these are derived from the $4 \times 4$ submatrix of the $6 \times 6$ orbit matrix given below. If the (symplectic) $4 \times 4$ matrix is of the form

\begin{tabular}{|l|l|}
\hline$M$ & $n$ \\
\hline$m$ & $N$ \\
\hline
\end{tabular}

where $M, N, m, n$ are $2 \times 2$ submatrices, then the coupling arises from the off-diagonal submatrices $\mathrm{m}$ and $\mathrm{n}$. The normal modes $\underline{\underline{u}}$ and $\underline{\mathrm{v}}$ are related to the geometric coordinates $\underline{\mathrm{x}}$ and $\underline{\mathrm{y}}$ by

4

$$
\underline{\mathrm{x}}=\underline{\mathrm{u}} \cos \varphi+\mathrm{D} \underline{\mathrm{v}} \sin \varphi ; \underline{\mathrm{y}}=-\mathrm{D}^{\prime} \underline{\mathrm{u}} \sin \varphi+\underline{\mathrm{v}} \cos \varphi
$$

where $\varphi$ may be called the "coupling angle" and D is a certain symplectic $2 \times 2$ matrix. The angle $\varphi$ and the matrix $\mathrm{D}$ can be obtained from the matrices $M, m, n, N$. Full coupling corresponds to $\varphi=45$ degrees

The "minimum tune separation", i.e. the tune splitting if the uncoupled tunes are exactly at resonance, is

$$
\Delta v=\left(\operatorname{Det}\left(n+m^{\prime}\right)\right)^{1 / 2} /(2 \pi)
$$

${ }^{1}$ T Roser et al, Spin Note No. 72, 1998

${ }^{2}$ E D Courant, Spin Note No. 83, 1999

${ }^{3}$ E D Courant, Spin Note No. 4 (1996) 
where $m^{\prime}$ is the symplectic conjugate of $m$.

This quantity $\Delta v$ is a better measure of the coupling strength than the coupling angle, since it is independent of how close one is to resonance, while the coupling angle will always be $45^{\circ}$ exactly at resonance no matter how weak the coupling is, as long as it is not zero.

Below we tabulate the matrices at $\mathrm{C} 10$ for AGS injection energy $(1.5 \mathrm{GeV})$, for the energies of the important intrinsic resonances $0+\nu_{y}$ and $36-\nu_{y}$, and for the transfer energy $\gamma \mathrm{G}=46.5$. We do this for the $5 \%$ solenoid snake, the helical snake, and the 4-dipole snake described above. We also show the orbit excursions inside the snake for the helical and 4-dipole case. (in the solenoid case the orbit displacement is zero). The matrices are similar to those previously obtained by N. Tsoupas and M. Okamura.

Note that the coupling parameters with a solenoid are independent of energy. The coupling effects in the helix and 4-dipole cases decrease approximately with the square of the energy (not quite, because the helical or dipole fields have to increase a bit with energy because $(1+\gamma G)$ increases a little faster than $\beta \gamma$ ). At injection energy the coupling in the 4-dipole case is distinctly stronger than for the solenoid (this is due to the focusing effect at the ends of the dipoles, where the excursions are quite large up to $5 \mathrm{~cm}$ ). The helix has an order of magnitude weaker coupling, and exhibits less coupling than the solenoid even at injection energy. At the higher energies - $\gamma \mathrm{G}=27$ to $46-$ the coupling is very small in both the helix and dipole cases.

We see that the helix is definitely preferable - if it is practicable.

\section{AGS without snakes}

\begin{tabular}{|r|r|r|r|r|r|}
\hline-1.31324 & -11.2107 & 0 & 0 & 0 & 2.08681 \\
\hline 0.167845 & 0.671359 & 0 & 0 & 0 & -0.318092 \\
\hline 0 & 0 & 1.57424 & -19.7498 & 0 & 0 \\
\hline 0 & 0 & 0.177118 & -1.58682 & 0 & 0 \\
\hline-0.06747 & -2.16502 & 0 & 0 & 1. & -11.5419 \\
\hline 0 & 0 & 0 & 0 & 0 & 1. \\
\hline
\end{tabular}

Tunes $\{8.698,8.749\}$

Matrix with solenoid snake

\begin{tabular}{|r|r|r|r|r|r|}
\hline-1.31093 & -11.1934 & 0.0442375 & -0.554929 & 0 & 2.08299 \\
\hline 0.168355 & 0.675285 & 0.00497101 & -0.0444856 & 0 & -0.318932 \\
\hline 0.0368755 & 0.31486 & 1.57265 & -19.7279 & 0 & -0.0585927 \\
\hline-0.0047357 & -0.0189952 & 0.176721 & -1.58147 & 0 & 0.0089713 \\
\hline-0.06747 & -2.16502 & 0 & 0 & 1. & -11.5419 \\
\hline 0 & 0 & 0 & 0 & 0 & 1. \\
\hline
\end{tabular}

Minimum tune split $=0.0144566$; coupling angle 8.08129

Tunes $\{8.69744,8.75032\}$ 


\section{Injection: $\gamma \mathrm{G}=4.65904$}

\section{Helical Snake:}

Matrix with helical snake

\begin{tabular}{|r|r|r|r|r|r|}
\hline-1.23141 & -10.5775 & -0.0130737 & 0.16348 & 0 & 1.8941 \\
\hline 0.220509 & 1.08209 & -0.00114092 & 0.00848222 & 0 & -0.403393 \\
\hline-0.0106962 & -0.0918811 & 1.50175 & -18.7783 & 0 & 0.0163952 \\
\hline 0.00194014 & 0.0095958 & 0.128475 & -0.940651 & 0 & -0.00375087 \\
\hline-0.0790867 & -2.21748 & 0.000304394 & -0.00384412 & 1. & -11.5189 \\
\hline 0 & 0 & 0 & 0 & 0 & 1. \\
\hline
\end{tabular}

Minimum tune split $=0.00448604$; coupling angle 2.26851

tunes $\{8.73802,8.79535\}$

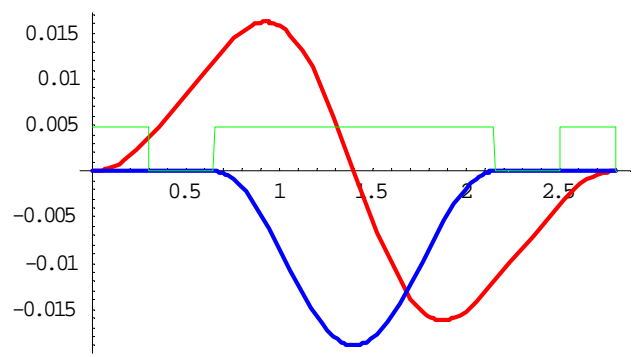

4-Dipole Snake

Matrix with 4-dipole snake

\begin{tabular}{|r|r|r|r|r|r|}
\hline-1.16851 & -10.0816 & -0.0159327 & 0.18963 & 0 & 1.84956 \\
\hline 0.243827 & 1.2475 & -0.0351435 & 0.44673 & 0 & -0.443225 \\
\hline 0.00648536 & 0.0692911 & 1.51749 & -19.0392 & 0 & -0.00780593 \\
\hline 0.0301393 & 0.252109 & 0.12114 & -0.860595 & 0 & -0.0469726 \\
\hline-0.0668694 & -2.1598 & -0.00172722 & 0.0227037 & 1. & -11.5333 \\
\hline 0 & 0 & 0 & 0 & 0 & 1. \\
\hline
\end{tabular}

Minimum tune split $=0.0539474 ;$ Coupling angle $=24.7769$

tunes $\{8.74383,8.81666\}$

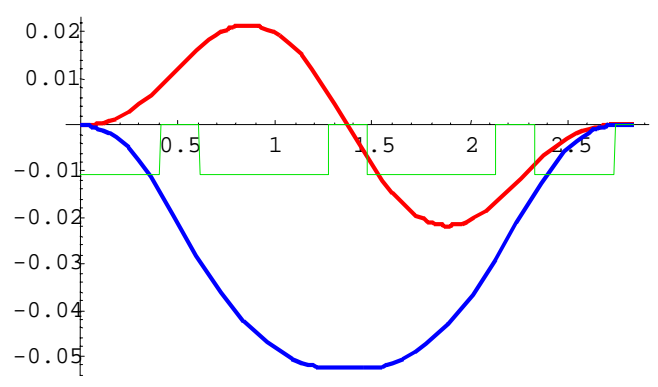


$\gamma \mathrm{G}$ at resonance $0+v=8.749$ :

Helical Snake

Matrix with helical snake,

\begin{tabular}{|r|r|r|r|r|r|}
\hline-1.2856 & -10.9968 & -0.00454085 & 0.0569063 & 0 & 2.00705 \\
\hline 0.185674 & 0.810388 & -0.000473248 & 0.00404146 & 0 & -0.347115 \\
\hline-0.00376408 & -0.0321978 & 1.54975 & -19.4215 & 0 & 0.00586524 \\
\hline 0.000546455 & 0.00239514 & 0.160639 & -1.36787 & 0 & -0.00106141 \\
\hline-0.0735955 & -2.1907 & 0.0000610821 & -0.000772655 & 1. & -11.5299 \\
\hline 0 & 0 & 0 & 0 & 0 & 1. \\
\hline
\end{tabular}

Minimum tune split $=0.00151209$; coupling angle 0.828196 tunes $\{8.71181,8.7645\}$

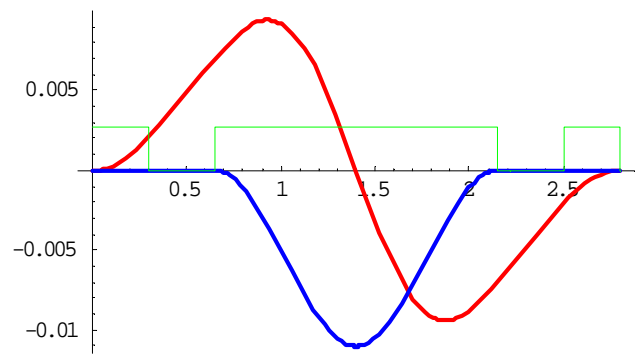

4- dipole snake

Matrix with 4-dipole snake

\begin{tabular}{|r|r|r|r|r|r|}
\hline-1.27088 & -10.8553 & -0.00534599 & 0.0635777 & 0 & 2.01906 \\
\hline 0.193523 & 0.866087 & -0.0118967 & 0.151276 & 0 & -0.360449 \\
\hline 0.00252978 & 0.0259674 & 1.54818 & -19.4481 & 0 & -0.00342083 \\
\hline 0.0106925 & 0.0890585 & 0.158157 & -1.34081 & 0 & -0.0168911 \\
\hline-0.0673455 & -2.16395 & -0.000337626 & 0.00443806 & 1. & -11.5389 \\
\hline 0 & 0 & 0 & 0 & 0 & 1. \\
\hline
\end{tabular}

Minimum tune split $=0.0186456$; Coupling angle $=10.4723$ tunes $\{8.7158,8.76827\}$

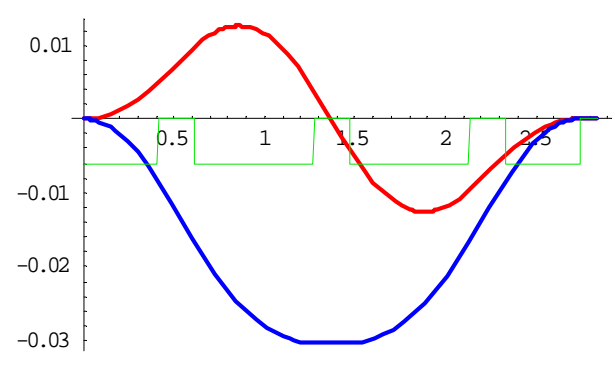


$\gamma \mathrm{G}$ at resonance $36-\mathrm{v}=27.251:$

Helical snake

Matrix with helical snake,

\begin{tabular}{|r|r|r|r|r|r|}
\hline-1.30994 & -11.1852 & -0.000547999 & 0.00687408 & 0 & 2.06946 \\
\hline 0.169973 & 0.687949 & -0.00006112 & 0.000544763 & 0 & -0.321571 \\
\hline-0.000456803 & -0.00390049 & 1.57132 & -19.7106 & 0 & 0.000721203 \\
\hline 0.0000593128 & 0.000240219 & 0.175151 & -1.56068 & 0 & -0.000113869 \\
\hline-0.0694881 & -2.17314 & 0.0000025 & -0.000032135 & 1. & -11.538 \\
\hline 0 & 0 & 0 & 0 & 0 & 1. \\
\hline
\end{tabular}

Minimum tune split $=0.000179397$; coupling angle 0.102084

tunes $\{8.69967,8.75085\}$

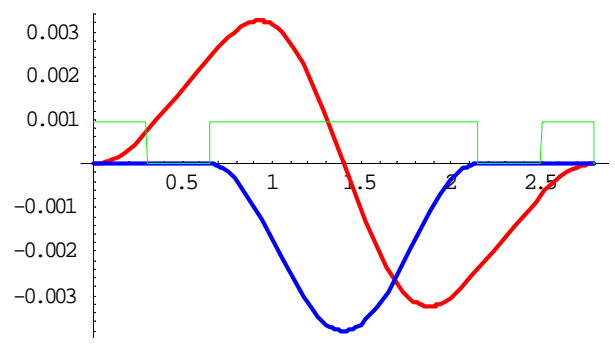

4-dipole snake

Matrix with 4-dipole snake

\begin{tabular}{|r|r|r|r|r|r|}
\hline-1.31693 & -11.2033 & -0.000635388 & 0.00755368 & 0 & 2.09529 \\
\hline 0.170907 & 0.694581 & -0.0014196 & 0.018054 & 0 & -0.32315 \\
\hline 0.000319777 & 0.00323311 & 1.56192 & -19.6312 & 0 & -0.000461704 \\
\hline 0.00130226 & 0.0108269 & 0.174855 & -1.55745 & 0 & -0.00207864 \\
\hline-0.0674648 & -2.16498 & -0.00001387 & 0.000182324 & 1. & -11.5415 \\
\hline 0 & 0 & 0 & 0 & 0 & 1. \\
\hline
\end{tabular}

Minimum tune split $=0.00224526$; Coupling angle $=1.28866$ tunes $\{8.69961,8.75038\}$

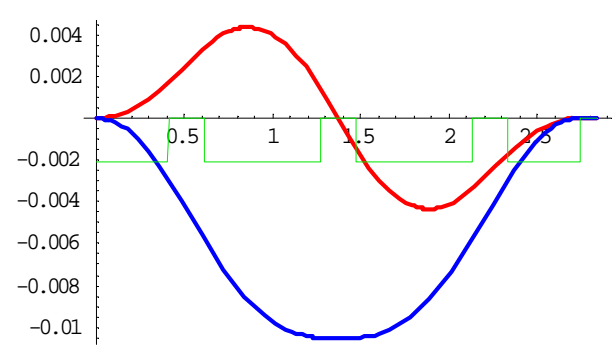




\section{$\gamma \mathrm{G}$ at transfer to $\mathrm{RHIC}=46.5$ :}

Helical snake

Matrix with helical snake,

\begin{tabular}{|r|r|r|r|r|r|}
\hline-1.31207 & -11.2017 & -0.000194072 & 0.00243463 & 0 & 2.07779 \\
\hline 0.168598 & 0.677229 & -0.000021768 & 0.000194669 & & 0 \\
\hline-0.000161853 & -0.0013818 & 1.57321 & -19.7359 & -0.319324 \\
\hline 0.0000208027 & 0.0000835805 & 0.176422 & -1.57757 & 0 & 0.000256213 \\
\hline-0.0686653 & -2.16981 & 0.0000005 & -0.00000677 & 0 & -0.0000397 \\
\hline 0 & 0 & 0 & 0 & 1. & -11.5396 \\
\hline
\end{tabular}

Minimum tune split $=0.0000634312$; coupling angle 0.0362184

tunes $\{8.69859,8.74965\}$

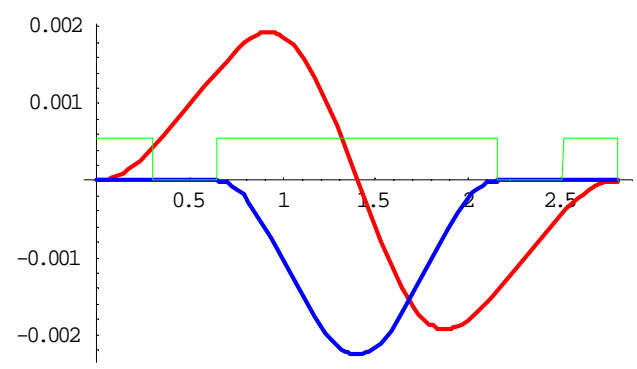

4-dipole snake

Matrix with 4-dipole snake

\begin{tabular}{|r|r|r|r|r|r|}
\hline-1.32096 & -11.2337 & -0.000224722 & 0.00267147 & 0 & 2.10196 \\
\hline 0.168928 & 0.679575 & -0.000502253 & 0.00638759 & & 0 \\
\hline 0.000113691 & 0.00114803 & 1.56312 & -19.6472 & -0.319882 \\
\hline 0.000461554 & 0.00383676 & 0.176317 & -1.57643 & 0 & -0.00016606 \\
\hline-0.0674689 & -2.16501 & -0.0000029 & 0.0000383578 & 0 & -0.000738246 \\
\hline 0 & 0 & 0 & 0 & 1. & -11.5417 \\
\hline
\end{tabular}

Minimum tune split $=0.000794997$; Coupling angle $=0.455641$

tunes $\{8.69804,8.74894\}$

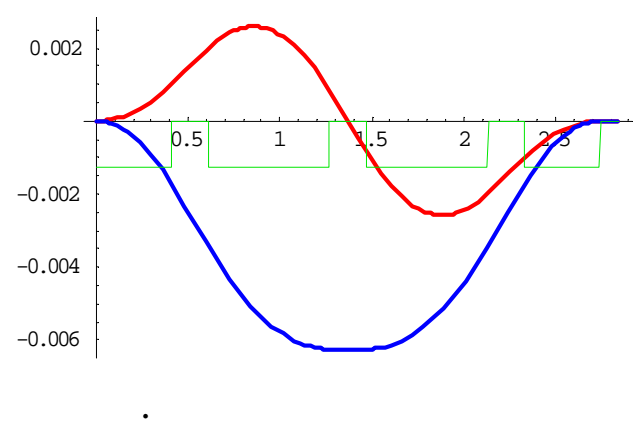

